\title{
Hypothyroidism symptoms in LT4-euthyroid thyroidectomized patients reflect an alteration of the serum proteome
}

Claudia Landi ( $\square$ landi35@unisi.it)

University of Siena

\section{Silvia Cantara}

University of Siena

Enxhi Shaba

University of Siena

Carlotta Marzocchi

University of Siena

Lorenza Vantaggiato

University of Siena

\section{Fabio Maino}

University of Siena

Alfonso Carleo

Hannover Medical School

Fabrizio Di Giuseppe

University of Chieti-Pescara

Stefania Angelucci

University of Chieti-Pescara

Luca Bini

University of Siena

Maria Grazia Castagna

University of Siena

\section{Research Article}

Keywords: Proteomics, hypothyroidism, reduced FT3, LT4, quality of life

Posted Date: April 1st, 2021

DOI: https://doi.org/10.21203/rs.3.rs-361476/v1 
License: (c) (i) This work is licensed under a Creative Commons Attribution 4.0 International License. Read Full License 


\section{Abstract}

In patients undergoing thyroidectomy one of the goals of post-surgical follow-up is the correct maintenance of thyroid hormone (TH) levels by replacement therapy with levo-thyroxine (LT4). Nevertheless, some patients complain of symptoms of hypothyroidism (memory loss, weight gain, fatigue, depression, and reduced quality of life) despite values of thyroid hormones get the physiological range.

Since this recurrent issue, we aim to compare serum proteomic profiles of LT4-euthyroid thyroidectomized patients with good or reduced post-operative quality of life.

Proteomic analysis highlights a different protein profile in the serum of patients with reduced quality of life compared to the other although both resulted euthyroid. Differential proteins are involved in coagulation processes, complement system activation and in lipoprotein particles remodeling, that together lead to a pro-inflammatory response. Moreover, a particular degradome of apolipoprotein A1, thrombin heavy chain and alpha 1 antitrypsin, was also detected.

This study suggests that LT4 replacement therapy might restore euthyroid conditions in thyroidectomized patients but in some cases without re-establish body tissues euthyroidism and serum protein profiles strongly suggest a behavior similar to overt hypothyroidism.

\section{Introduction}

Patients undergoing thyroidectomy need to maintain correct thyroid hormone $(\mathrm{TH})$ levels by replacement therapy with levo-thyroxine (LT4) ${ }^{1}$. The rationale for the use of LT4 is supported by the fact that the peripheral conversion desiodase-mediated of $\mathrm{T} 4$ into $\mathrm{T} 3$ is able to guarantee a correct homeostasis of THs in target tissues ${ }^{2}$. In the majority of patients, LT4 therapy is effective. However, in a subgroup of thyroidectomized subjects ${ }^{3,4}$, typical symptoms of hypothyroidism persist with a reduced quality of life (QoL) despite normalization of serum TSH levels.

We recently characterized a subgroup of patients with post-operative FT3 levels statistically significant lower, although in the normal range, than that observed before surgery submitted to total thyroidectomy and undergoing LT4 therapy. These patients complain symptoms of hypothyroidism even though their TSH levels were in the normal range and similar to those observed in the pre-surgical evaluation ${ }^{5}$. In our previous report [5] we concluded that this effect was linked to the DIO2 Thr92Ala polymorphism, but in the clinical practice, we saw that not all the patients with impaired QoL despite normal TSH and LT4therapy, carry the DIO2-variant, suggesting the involvement of other molecular mechanisms. These altered molecular mechanisms could be evaluated at the serum level and proteomics represents a promising tool to be applied. Little is known about the relationship between TH levels and circulating proteome profile. In literature, only few studies have evaluated the changes in circulating proteome in patients undergoing LT4 replacement therapy ${ }^{6}$. Among these, two papers reported plasma proteomics 
analyses in an experimentally induced human thyrotoxicosis model ${ }^{7,8}$. Engelmann et al., explored the effects of LT4 excess and found a positive correlation between FT4 levels and 10 proteins involved with blood coagulation ${ }^{7}$. Pietzneret al. detected 63 proteins significantly associated with serum FT4 levels. Among these proteins, complement and coagulation proteins were increased and apoliproteins were decreased ${ }^{8}$. Only one study explored plasma proteome changes that occur in the transition from hypothyroid to euthyroid state in patients during LT4-therapy ${ }^{9}$. This comparison revealed alterations of proteins involved in acute phase response highlighting that LT4 reduces pro-inflammatory cytokine levels. No studies took into consideration the effects of LT4 on circulating proteome in thyroidectomized patients and in particular in the subgroup of patients with normal TSH values but persisting symptoms of hypothyroidism.

Accordingly to our previous results ${ }^{5}$, we hypothesized that the subgroup of LT4-euthyroid thyroidectomized patients with impaired quality of life, could be associated with tissue hypothyroidism. To explore this hypothesis, serum proteomic profile were compared in postsurgical patients subjected to replacement with LT4-therapy with unchanged or reduced FT3 in the physiological range associated to an impaired quality of life.

\section{Results}

\section{Comparison of post-surgical thyroid hormone levels between "reduced" and "unchanged" FT3 patients}

Based on our previous report [5], we selected from the group of patients with "reduced" or "unchanged" FT3, 4 patients/group to submit to proteomic analysis. The "reduced" patients were chosen in order to have subjects complaining lower physical and neurocognitive wellbeing after total thyroidectomy. As shown in Table 1, there was no significant difference between post-surgery serum TSH and FT4 levels among groups (by Wilcoxon test for paired data). The mean post-surgical serum FT3 level was significantly $(p=0.05)$ lower in the "reduced" group compared to the "unchanged" $(3.4 \pm 0.5 \mathrm{pg} / \mathrm{ml}$ versus $2.7 \pm 0.15 \mathrm{pg} / \mathrm{ml}$ ) although in the reference range. Age, FT3/FT4 ratio, LT4 dose/day and LT4 dose/ $\mathrm{kg}$ did not differ between patients with a lower FT3 level after surgery and those in whom FT3 was unchanged. Due to the low number of patients, sex distribution was by chance different in the two groups.

\section{Differential proteomic results}

The analyzed dataset enumerated $\sim 1200$ protein spots. The unsupervised analysis (data not shown), performed as data quality control, was obtained analyzing the top 100 spots with higher variance intensity through unsupervised heatmap and PCA and the results did not highlight any outliers nor clustering related with any possible covariant disturbances.

However, the comparison emphasized 31 significant differentially abundant spots (DAS) between Reduced FT3 and unchanged FT3 groups (Table S1, Figure 1).

The 31 differential spots are visualizable in the reference gel maps in Figure 2 and Figure S-1. 


\section{Peptide Mass Fingerprint identification highlights a characteristic pattern of THRB, APOAI and A1AT protein fragments in FT3 reduced patients}

Once performed protein identification by MALDI-ToF mass spectrometer, Mascot Search Results of all differentially abundant spots were reported in Table 2. Interestingly, some identifications highlighted not the full-length protein but a protein fragment. In particular, spots $1 \mathrm{Q}, 2 \mathrm{Q}, 3 \mathrm{Q}$, visible only in reduced FT3 samples, were identified by Peptide Mass Fingerprint (PMF) as C-term fragments of thrombin from aa 364 to 622 and corresponding to thrombin heavy chain isoform on UniProt database (Figure 3), demonstrating a possible specific proteolytic process.

Among highly abundant spots in reduced FT3 sera we found spots 4, 11, 12, 21 and 22 . All are identified as full-length APOAl, even if spots 11 and 12 showed a mixture with a central fragment of A1AT suggesting a colocalization of these two protein species on the 2DE map (Table 2, Figure 4 and 5). Among low abundant spots in reduced FT3 sera there were spots 24,14 and $5 Q$, identified respectively as central, N-terminal and C-terminal fragments of APOAI (Figure 4). Two-dimensional western blot (2D WB) results shown in Figure 4 confirmed the abundance and the distribution of APOAl protein species with a higher abundance of full-length APOAl in spots 4, 11, 12, 21, 22 and a lower abundance of the protein fragments only in spot 24 in FT3 reduced condition.

At the same time, we were interested to validate A1AT proteomic data as well. While the highly abundant spot 15 in reduced FT3 samples was identified as A1AT full-length, spots 11, 12, 24 were identified as a mixture of a central fragments of A1AT and APOAI (Table 2; Figure 5, PMF identification), making impossible to evaluate A1AT abundance for these spots on 2DE. To overcome this problem, we applied a 2D WB approach using A1AT antibodies to validate and quantify A1AT protein pattern (Figure 5). The results corroborated the identification of spots 11 and 12 as A1AT and permitted to highlight differential abundance of A1AT fragments revealing an opposite trend with respect to APOAI. Spot 24 identified by PMF as a mix of APOAI and fragment of A1AT (Table 2; Figure 5, PMF identification), was confirmed by 2DWB also as A1AT (Figure 5) and low abundant in reduced FT3 samples.

\section{PCA and heatmap analysis}

The PCA carried on the 31 DASs summarized the $95.6 \%$ (PC1:85.2\% and PC2:10.4\%) of the variance and the two groups clusterized alongside the first component (Figure 6A). In addition, Figure 6B showed the contributions of each significant variant in the first two PCs highlighting spot 11, 12 and 22, identified as APOAl and fragments of A1AT, as the most significant. Similarly, to PCA, the heatmap branching separated the samples in two clusters corresponding to FT3 groups (Figure 6C).

\section{Enrichment analysis}

Enrichment analysis by MetaCore permitted the building of the network of molecular interactions related to the identified proteins. Figure 7 shows the protein network where thrombin, alpha1-antitrypsin, APOE, Plasminogen and APOA1 were central functional hubs i.e proteins with a higher number of interactions 
with respect to the other proteins. Light blue lines in the interactome show the canonical paths delineated in Table S-2 that also reports their relative GO biological processes. Three principal molecular paths such as blood coagulation (yellow), complement system (orange) and lipoprotein particle remodeling (blue) are evident.

Results by process network analysis showed that the inflammation, induced by complement system, Kallikrein-kinin system, IL-6 signaling, protein C signaling and innate inflammatory response, represents the central process where the identified proteins are involved (Table 3). Indeed, all three molecular paths highlighted on the protein network in Figure 7 suggest the activation of different pro-inflammatory ways.

Pathway Maps analysis highlights a strong involvement of the complement, blood coagulation and lipid particle remodeling with the terms "immune response by classical, alternative, lectin induced complement pathways", "Alternative complement cascade disruption in age-related macular degeneration" and "Complement pathway disruption in thrombotic microangiopathy", "blood coagulation" and terms such as "lipid metabolism", "HDL-mediated reverse cholesterol transport" and "HDL dyslipidemia in type 2 diabetes and metabolic syndrome X" (Table 4). Enrichment analysis by GO biological processes in Table S-3, strongly support the involvement of lipoproteins metabolism and remodeling.

\section{Discussion}

Patients undergoing thyroidectomy are normally treated with LT4 replacement therapy but a substantial proportion of them experience hypothyroid-like symptoms despite normal TSH levels. During LT4 replacement, levels of the active hormone FT3 was reported to be in the target range, only showing a slight inflection. Our previous study reported that thyroidectomized patients carrying Thr92Ala polymorphism are at increased risk of reduced intracellularand serum FT3 concentrations ${ }^{5,10}$ but these results did not explain completely as some patients, that complain symptoms of hypothyroidism, do not report such polymorphism. To this purpose, we were interested to study serum protein profiles of these patients in order to understand if, to peripheral level, there were characteristics able to justify hypothyroidlike symptoms. To this purpose, we perform a preliminary differential proteomic analysis of serum samples in FT3 "reduced" and FT3 stable patients that highlights a differential protein pattern between the two conditions. In particular, following enrichment analysis results, these altered proteins participate in processes such as blood coagulation, complement activation and lipoprotein particles remodeling. All these processes induce a proinflammatory response that could be retained responsible of hypothyroidism symptoms. In particular, blood coagulation suggests a proinflammatory response by the dysregulation of thrombin, plasminogen and alpha 1 antitrypsin, while complement activation can participate by altered $\mathrm{C} 3, \mathrm{C} 1 \mathrm{q}, \mathrm{C} 1 \mathrm{~s}$ and $\mathrm{CFAH}$ levels that we found. In accordance with our results, Bitencourtet al indicated that there is a connection between the immune system and thyroid hormones, sinceT3 and T4 hormones affect the lytic potency of the complement system ${ }^{11}$. In particular, we found an up-regulation of fragments that could be generated during the classical, alternative or lectin induced activation of the complement and that have important biological functions, including facilitation of phagocytosis, 
clearance of immunocomplexes, inflammation, immuneresponse, and tissue homeostasis ${ }^{12,13}$. Indeed, our process network analysis suggests that dysregulated proteins involved in blood coagulation and complement system such as $\mathrm{CFAH}, \mathrm{C} 3, \mathrm{C} 1 \mathrm{~s}$, Thrombin, Plasminogen, Alpha 1-antitrypsin, are responsible of inflammatory processes both by complement activation, kallikrein-kinin system (KKS) and IL-6 signaling. Complement and KKS are reported to be activated during vascular inflammation ${ }^{14}$ and their extensive activation on the endothelium promotes thrombosis, leukocyte recruitment, vascular permeability and vascular wall injury ${ }^{11,14}$. Basically, the complement, coagulation and fibrinolytic system lead to thrombo inflammation which collectively lead to the activation of blood cells such as polymorphonuclear cells, monocytes, platelets and endothelial cells (EC) lining the vessel ${ }^{15}$. Therefore, altered coagulation processes and complement system activation that we found, could suggest the presence of a potential vascular inflammation in FT3 "reduced" patients as already reported in subclinical hypothyroidism ${ }^{16}$. Of interest, different studies associate higher serum levels of inflammatory molecules to the fatigue and migraine onset, a characteristic symptoms of hypothyroidism ${ }^{17-19}$ as well as to depression ${ }^{20}$. Indeed, inflammation related to a potential endothelial dysfunction, as suggested by our data, was already reported in depression ${ }^{21}$ and may prove to be the cause for this illness also in FT3 reduced patients.

The role of thrombin and $\mathrm{A} 1 \mathrm{AT}$ as central hubs of our protein network makes them potential biomarkers of hypothyroidism symptoms despite values of TSH in the reference range. Moreover, their characteristic protein fragmentation that we found, suggests a protein impairment in FT3 reduced serum that could be responsible and peculiar for this condition. These diverse patterns of alpha1-antitrypsin fragment simply different cleavage pathways occurring after thyroidectomy and FT4 replacement. Since the peculiar roles of serpins in regulating proteases of plasma, mostly enzymes of blood coagulation, complement, and inflammatory systems ${ }^{22}$, the impaired fragmentation of A1AT, furtherly suggest an alteration of these pathways in LT4-euthyroid thyroidectomized patients with reduced quality of life.

Thrombin fragments, that we identified only in FT3 reduced patients, represent the heavy chain of the protein, a carboxy-terminal fragment known as serineprotease domain ${ }^{23}$. The presence of A1AT and THRB fragments not only reports to an alteration in coagulation processes but also to a serine protease/serpins impairment, potential cause of hypothyroidism symptoms. Chadarevian R et a/ reported that plasma levels of some components of the fibrinolytic system correlated to plasma levels of T4 24 suggesting a possible correlation between FT4 replacement therapy modulation and coagulation cascade.

Another interesting finding of our study regards the lipoprotein particle remodeling pathway alteration suggested by the dysregulation of APOA4, APOAI, APOE, albumin and haptoglobinBecause thyroid hormone influences all aspects of lipid metabolism such as synthesis, mobilization, and degradation, dyslipidemia is very common in thyroid disease ${ }^{25}$. In this case, APOAI and APOE, up-regulated in FT3 reduced patients in agreement with data reported by 0 'Brien et $a^{26}$, resulted central functional hubs of our analysis suggesting their potential role as biomarkers. In particular, our experiments highlight an high 
abundance of the APOAl full-length and simultaneously, a decrease of APOAI central, N-terminal and Cterminal fragments with respect to FT3 stable condition, accordingly with Jung et $a R^{25}$, and demonstrating a specific resistance to proteolysis mechanisms. Dysregulation of APOAI management could suggest a potential lipoprotein particle dysfunction. To this purpose, Jung et al also report that increased HDL-cholesterol levels with impaired function persisted despite restoration of thyroid hormone levels and that the concentration of APOE showed a similar pattern to that of HDL-cholesterol ${ }^{25}$ in agreement with our results. Changes in thyroid function are associated not only with changes in the concentrations of various plasma lipid components but also with changes in HDL functions ${ }^{25}$ and our results suggest a possible dysfunction in lipoprotein particle remodeling.

In light with our findings, enrichment analysis confirms that the above-mentioned pathways induce proinflammatory responses by complement system activation but also by kallikrein-kinin system and by IL- 6 signaling according to Zhou et $a^{27}$ that reported an increasing of IL- 6 and TNF-a after hypothyroidism induction in rats. Together, these results suggest an interrelationship between inflammation and hormone derangement ${ }^{28}$, probably establishing a vicious circle reflected in the impaired quality of life of "FT3 reduced" patients at physical and neurocognitive levels.

\section{Materials And Methods}

\section{Patients' population}

Patients were selected from the study population evaluated in a previous published study included patients submitted to total thyroidectomy. Inclusion criteria were: 1) thyroid profile data obtained within 10 months of surgery; 2) post-surgical thyroid profile obtained at least 6 months after achievement of a stable thyroid hormone status on LT4 therapy; 3 ) pre- and post-surgical serum TSH levels not different by more than $\pm 0.5 \mathrm{mIU} / \mathrm{L}$. Patients with an abnormal thyroid profile (hypo- or hyperthyroidism) before surgery, patients receiving drugs that can interfere with thyroid function, and patients affected by malabsorption-related conditions were excluded.

We arbitrarily selected a change of at least $0.5 \mathrm{pg} / \mathrm{ml}$ as a significant variation between pre- and postsurgical FT3 value. According to this criterion, patients were classified as having "reduced FT3" when post-surgical FT3 levels were at least $0.5 \mathrm{pg} / \mathrm{ml}$ lower than pre-surgical FT3 values. In all patients FT3 levels were into the normal range before and after surgery and no difference between pre- and postsurgical TSH levels were observed [5]. From our previous cohort we selected postsurgical sera of 8 patients (4 patients with "reduced FT3" and 4 with "unchanged FT3"). In the "reduced" group we included only patients complaining with lower physical and neurocognitive wellbeing after surgery despite target TSH levels. The study cohort included $62.5 \%$ of females with a mean age of $61.4 \pm 13.8$ years (range 3780 years) and with thyroid nodular disease. At final histology, 7/8 (87.5\%) patients had differentiated thyroid carcinoma and $1 / 8(12.5 \%)$ had a benign goiter. Immediately after surgery, patients were treated with LT4 to obtain comparable pre-surgical TSH levels, with a mean dose of $114.9 \mathrm{ug} /$ day and a mean dose/kg of 1.54 ug of LT4.Fasting blood samples were collected at 08.00-09.00 hours before patients 
assumed the LT4 tablet, and all determinations were performed with a chemiluminescent immunometric assay (Access Immunoassay Systems 2006, Beckman Coulter, Milan, Italy). Normal ranges were 2.5-4.5 $\mathrm{pg} / \mathrm{ml}$ for FT3, 5.8-16.4 pg/ml for FT4 and 0.4-4-0 mlU/L for TSH.

All methods and procedures were carried out in accordance with relevant guidelines and regulations. The study was approved by our local ethical committee "Office of Ethical Affairs. Azienda OspedalieroUniversitaria Senese". Informed consent was obtained from all subjects enrolled in the study.

\section{Proteomic and enrichment analysis}

Blood was collected directly into serum tubes (BD vacutainer, SST II Advance, Plymouth UK) and centrifuged for $10 \mathrm{~min}$ at $1690 \mathrm{xg}$. The serum was recovered and stored at $-80{ }^{\circ} \mathrm{C}$ until proteomic analysis. Samples were prepared and resolved by two-dimensional electrophoresis (2DE), according to Landi $C$ et $a^{29}$. MALDI-ToF mass spectrometry by Peptide Mass Fingerprint (PMF) was considered for protein identification ${ }^{30}$. The data analysis of relative percentage of spot volume was carried out using RStudio Desktop 1.1.463 (Integrated Development for RStudio, Inc., Boston, USA, https://www.rstudio.com). Firstly, a prelaminar unsupervised analysis focused on the most 100 variant spots as data quality control. The significant changes in protein abundance between the Reduce FT3 and Stable FT3 groups was obtained by limma tool employing linear models and empirical Bayesian methods $^{31,32}$. Spots presenting $p$-value lower than 0.05 were considered as significant and were submitted to Heatmap and Principal Component Analysis (PCA). In particular, spots and samples were clustered in the Heatmap according Ward's minimum variance method and Euclidean distance. The UniProt accession numbers of the identified proteins were used to perform enrichment analysis by MetaCore software (https://portal.genego.com) (Clarivate analytics, Boston, MA).

\section{Bidimensional Western Blot validation of A1ATand APOAI fragmentation}

After bidimensional electrophoresis separation, proteins were transferred onto nitrocellulose membrane (Hybond ECL, GE Healthcare) ${ }^{33-35}$. Ponceau Red staining (0.2\% w/v Ponceau S in 3\% v/v trichloroacetic acid) of the membranes was performed to verify correct protein transfer and to assess equal protein loading (data not showed). Western blot analysis was performed using: goat polyclonal antibodies against alpha 1 antitrypsin (Santa Cruz) andmouse monoclonal anti-apolipoprotein Al (Santa Cruz. Antigoat and anti-mouse secondary antibodies were purchased from Sigma Aldrich. All antibodies were diluted according to the manufacturer's instructions and chemioluminescent signals, obtained after using an ECL kit (GE Healthcare), were captured at different time-points of exposure. The digital 2-D western blot images were visualized by Image Master platinum 7.0.

\section{Conclusions}

Despite biochemical characteristics of subjects that report TSH and FT3 levels in the reference range during LT4 replacement therapy, some thyroidectomized patients, with FT3 reduced in the physiological range, complain symptoms of hypothyroidism. Comparing serum proteomes, we found that in FT3 
reduced patients, protein profiles strongly suggest a behavior similar to overt hypothyroidism with dysregulation of complement activation, blood coagulation and lipoprotein particle remodeling. Moreover, a characteristic APOAI, A1AT and THRB degradome was highlighted. Together these molecular ways sustain a pro-inflammatory response also by kallikrein-kinin system and by IL- 6 signaling that in part could suggest the hypothyroidism symptoms.

In conclusion, our results suggest that LT4 replacement therapy might not restore body tissues euthyroidism in all thyroidectomized patients, despite TSH levels is in the normal range. Further prospective studies, including a large cohort of patients, will be needed to confirm and amplify our results.

\section{Declarations}

\section{Acknowledgement}

We are grateful to MERCK for contributing to the work.

\section{Author contribution}

All authors had full access to all the data of the study and took responsibility for the integrity of the data and the accuracy of the data analysis. Conceptualization, Maria Grazia Castagna, Claudia Landi, Silvia Cantara; Formal analysis, Claudia Landi, Silvia Cantara and Carlotta Marzocchi; Investigation, Claudia Landi, Lorenza Vantaggiato and Alfonso Carleo; Methodology, Claudia Landi, Enxhi Shaba, Fabio Maino, Fabrizio Di Giuseppe and Stefania Angelucci; Writing - original draft, Claudia Landi and Silvia Cantara; Writing - review \& editing, Luca Bini and Maria Grazia Castagna.

\section{Conflict of interest}

Authors have nothing to declare

\section{References}

1. Garber, J. R. et al. Clinical practice guidelines for hypothyroidism in adults: cosponsored by the American Association of Clinical Endocrinologists and the American Thyroid Association. Endocr Pract 18, 988-1028 (2012).

2. Arrojo E Drigo, R., Fonseca, T. L., Werneck-de-Castro, J. P. S. \& Bianco, A. C. Role of the type 2 iodothyronine deiodinase (D2) in the control of thyroid hormone signaling. Biochim Biophys Acta 1830, 3956-3964 (2013).

3. Saravanan, P. et al. Psychological well-being in patients on 'adequate' doses of I-thyroxine: results of a large, controlled community-based questionnaire study. Clin Endocrinol (Oxf) 57, 577-585 (2002).

4. Wiersinga, W. M., Duntas, L., Fadeyev, V., Nygaard, B. \& Vanderpump, M. P. J. 2012 ETA Guidelines: The Use of L-T4 + L-T3 in the Treatment of Hypothyroidism. Eur Thyroid J 1, 55-71 (2012). 
5. Castagna, M. G. et al. DIO2 Thr92Ala Reduces Deiodinase-2 Activity and Serum-T3 Levels in ThyroidDeficient Patients. J Clin Endocrinol Metab 102, 1623-1630 (2017).

6. Pagni, F. et al. Proteome analysis in thyroid pathology. Expert Rev Proteomics 12, 375-390 (2015).

7. Engelmann, B. et al. Effect of Experimental Thyrotoxicosis onto Blood Coagulation: A Proteomics Study. Eur Thyroid J 4, 119-124 (2015).

8. Pietzner, M. et al. Plasma proteome and metabolome characterization of an experimental human thyrotoxicosis model. BMC Med 15, 6 (2017).

9. Alfadda, A. A., Benabdelkamel, H., Masood, A., Jammah, A. A. \& Ekhzaimy, A. A. Differences in the Plasma Proteome of Patients with Hypothyroidism before and after Thyroid Hormone Replacement: A Proteomic Analysis. Int J Mol Sci 19, (2018).

10. Cantara, S. et al. Variants in MCT10 protein do not affect FT3 levels in athyreotic patients. Endocrine 66, 551-556 (2019).

11. Bitencourt, C. S., Duarte, C. G., Azzolini, A. E. C. S. \& Assis-Pandochi, A. I. Alternative complement pathway and factor B activities in rats with altered blood levels of thyroid hormone. Braz J Med Biol Res 45, 216-221 (2012).

12. Afshar-Kharghan, V. The role of the complement system in cancer. J Clin Invest 127, 780-789 (2017).

13. Barrington, R., Zhang, M., Fischer, M. \& Carroll, M. C. The role of complement in inflammation and adaptive immunity. Immunol Rev 180, 5-15 (2001).

14. Lopatko Fagerström, I. et al. Blockade of the kallikrein-kinin system reduces endothelial complement activation in vascular inflammation. EBioMedicine 47, 319-328 (2019).

15. Ekdahl, K. N. et al. Dangerous liaisons: complement, coagulation, and kallikrein/kinin cross-talk act as a linchpin in the events leading to thromboinflammation. Immunol Rev 274, 245-269 (2016).

16. Türemen, E. E., Çetinarslan, B., Şahin, T., Cantürk, Z. \& Tarkun, I. Endothelial dysfunction and low grade chronic inflammation in subclinical hypothyroidism due to autoimmune thyroiditis. Endocr $J$ 58, 349-354 (2011).

17. Himbert, C. et al. Inflammation- and angiogenesis-related biomarkers are correlated with cancerrelated fatigue in colorectal cancer patients: Results from the ColoCare Study. Eur J Cancer Care (Engl) 28, e13055 (2019).

18. Herlofson, K. et al. Inflammation and fatigue in early, untreated Parkinson's Disease. Acta Neurol Scand 138, 394-399 (2018).

19. E, B. et al. Proteomic serum profile in menstrual-related and post menopause migraine. Journal of pharmaceutical and biomedical analysis vol. 184 https://pubmed.ncbi.nlm.nih.gov/32113117/ (2020).

20. Landi, C. et al. Cognitive impairment and CSF proteome modification after oral bacteriotherapy in HIV patients. J Neuroviro/ 26, 95-106 (2020). 
21. Halaris, A. Inflammation-Associated Co-morbidity Between Depression and Cardiovascular Disease. Curr Top Behav Neurosci 31, 45-70 (2017).

22. Schmaier, A. H. Serpin targets in hemostasis/kinin formation. Blood 134, 1566-1568 (2019).

23. Davie, E. W. \& Kulman, J. D. An overview of the structure and function of thrombin. Semin Thromb Hemost 32 Suppl 1, 3-15 (2006).

24. Chadarevian, R. et al. Components of the fibrinolytic system are differently altered in moderate and severe hypothyroidism. J Clin Endocrinol Metab 86, 732-737 (2001).

25. Jung, K. Y. et al. Association between thyroid function and lipid profiles, apolipoproteins, and highdensity lipoprotein function. J Clin Lipido/ 11, 1347-1353 (2017).

26. O'Brien, T. et al. The effect of the treatment of hypothyroidism and hyperthyroidism on plasma lipids and apolipoproteins Al, All and E. Clin Endocrinol (Oxf) 46, 17-20 (1997).

27. Zhou, J., Cheng, G., Pang, H., Liu, Q. \& Liu, Y. The effect of 131 l-induced hypothyroidism on the levels of nitric oxide (NO), interleukin 6 (IL-6), tumor necrosis factor alpha (TNF-a), total nitric oxide synthase (NOS) activity, and expression of NOS isoforms in rats. Bosn J Basic Med Sci 18, 305-312 (2018).

28. Mancini, A. et al. Thyroid Hormones, Oxidative Stress, and Inflammation. Mediators Inflamm 2016, 6757154 (2016).

29. Landi, C. et al. Ceruloplasmin and oxidative stress in severe eosinophilic asthma patients treated with Mepolizumab and Benralizumab. Biochim Biophys Acta Proteins Proteom 1869, 140563 (2021).

30. Sulpizio, M. et al. Molecular basis underlying the biological effects elicited by extremely lowfrequency magnetic field (ELF-MF) on neuroblastoma cells. J. Cell. Biochem. 112, 3797-3806 (2011).

31. Kammers, K., Cole, R. N., Tiengwe, C. \& Ruczinski, I. Detecting Significant Changes in Protein Abundance. EuPA Open Proteom 7, 11-19 (2015).

32. Ritchie, M. E. et al. limma powers differential expression analyses for RNA-sequencing and microarray studies. Nucleic Acids Res 43, e47 (2015).

33. Laemmli, U. K. Cleavage of structural proteins during the assembly of the head of bacteriophage T4. Nature 227, 680-685 (1970).

34. Towbin, H., Ozbey, O. \& Zingel, O. An immunoblotting method for high-resolution isoelectric focusing of protein isoforms on immobilized pH gradients. Electrophoresis 22, 1887-1893 (2001).

35. Towbin, H., Staehelin, T. \& Gordon, J. Electrophoretic transfer of proteins from polyacrylamide gels to nitrocellulose sheets: procedure and some applications. Proc Natl Acad Sci U S A 76, 4350-4354 (1979).

\section{Tables And Supplementary Materials}

Tables 1-4 are available in the Supplementary Files. 
Table 1. Comparison of post-surgical thyroid hormone levels between "reduced" and "unchanged" FT3 patients.

Table 2. Table reports Mascot search results by Score, number of matched peptides and sequence coverage. Spot numbers correspond to that in Figure 2 and Table S1. Moreover, Table also reports UniProtKB protein description, abbreviation, accession number (AC), theoretical pl and $\mathrm{MW}$ and the $\% \mathrm{~V}$ protein ratio in stable (S) and reduced (R) FT3 samples.

Table 3. Process networks by MetaCore software reporting p-values, FDR and network objects from active data.

Table 4. Pathway Maps by MetaCore software reporting $p$-values, FDR and network objects from active data.

\section{Supplemental table captions}

Table S1. Table reporting the 31 significant differentially abundant spots between Reduced FT3 and Unchanged FT3 groups. Spot numbers correspond to that of Table 1 and Figure 2. Moreover, are also reported UniProtKB protein description, abbreviation, accession number (AC), \%V mean and standard deviation in unchanged and reduced FT3 samples, p-values, F.p values and the \%V mean ratio between Stable and Reduced FT3.

Table S2. Canonical paths reported in the protein network in Figure 7 (edges highlighted in light blue). GO biological processes are reported for each canonical path together with $p$-values.

Table S3. GO biological processes enrichment analysis by MetaCore software. Table also reports pvalues, FDR and network objects from active data.

\section{Supplemental Figure captions}

Figure S1. Histograms of the 31 significant differentially abundant spots (DAS) between Reduced FT3 and Unchanged FT3 groups. In particular, the A section reports the highly abundant spots in reduced FT3 while the $B$ section reports the low abundant spots.

\section{Figures}




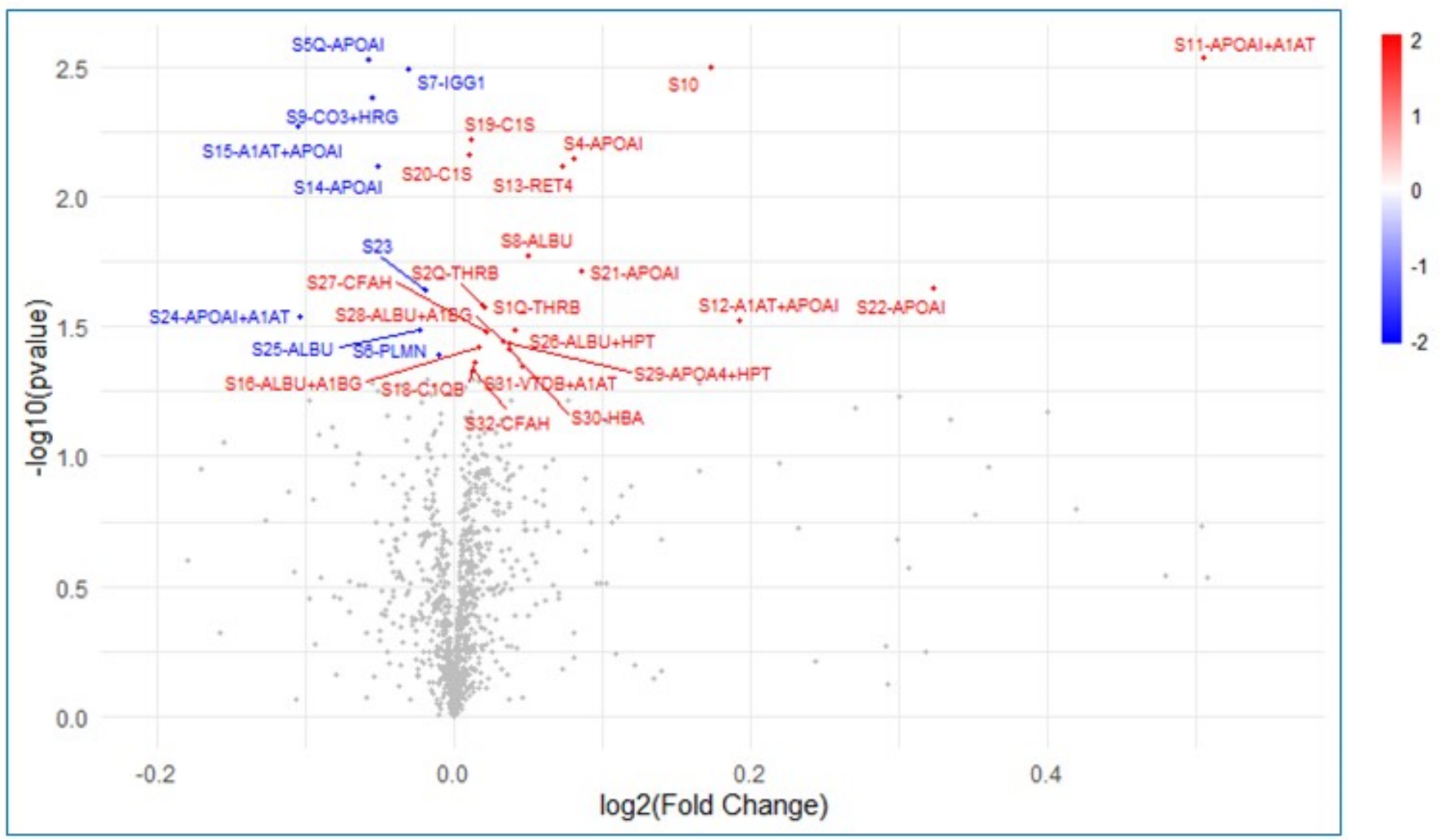

\section{Figure 1}

Figure reports the volcano plot emphasizing the 31 significant differentially abundant spots (DAS) between Reduced FT3 and Unchanged FT3 groups. Red dots and blue dots respectively represent upregulated and down-regulated proteins in reduced FT3 samples. 


\section{Reduced FT3}

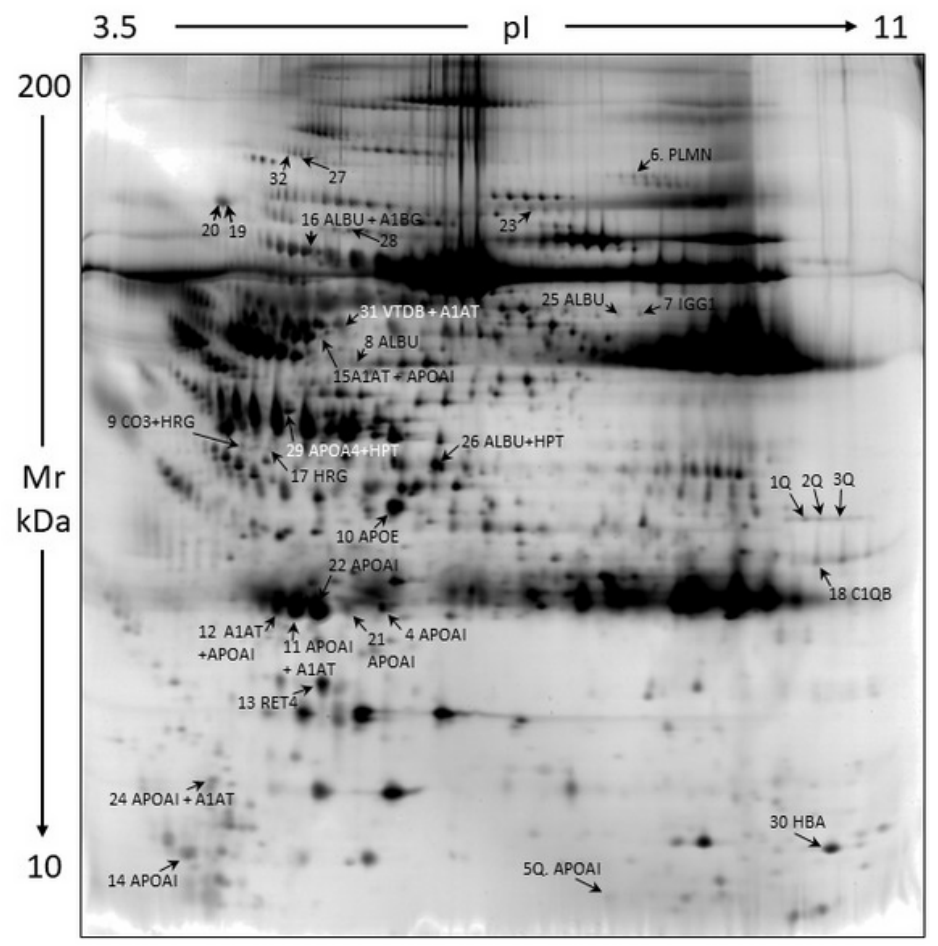

\section{Unchanged FT3}

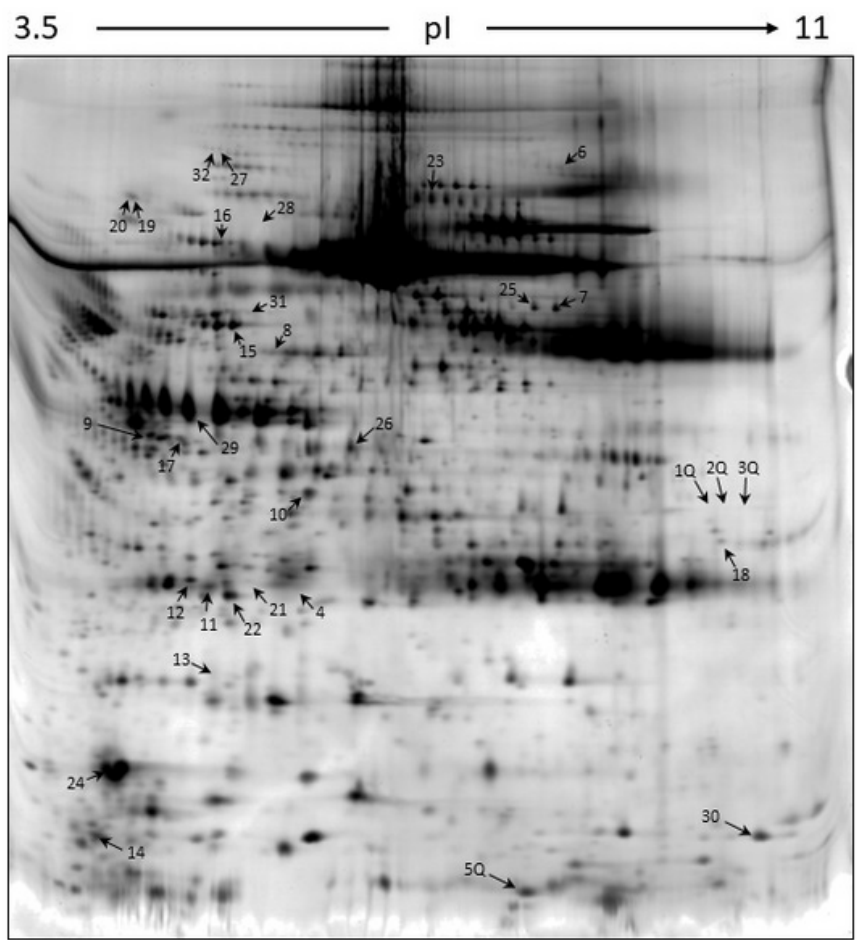

\section{Figure 2}

Electrophoretic maps of Reduced and Unchanged FT3 samples highlighting the differential spots found. Numbers correspond to that in Table 1 and Table S-1.

\section{Thrombin heavy chain}

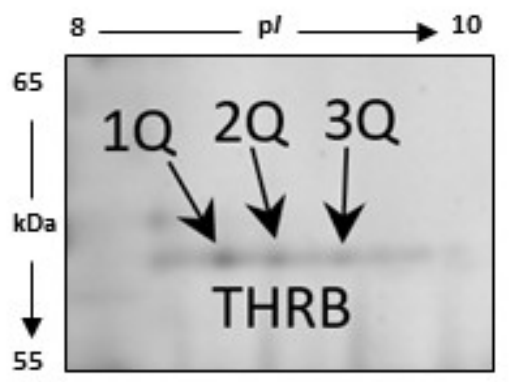

Matched peptides shown in bold red.

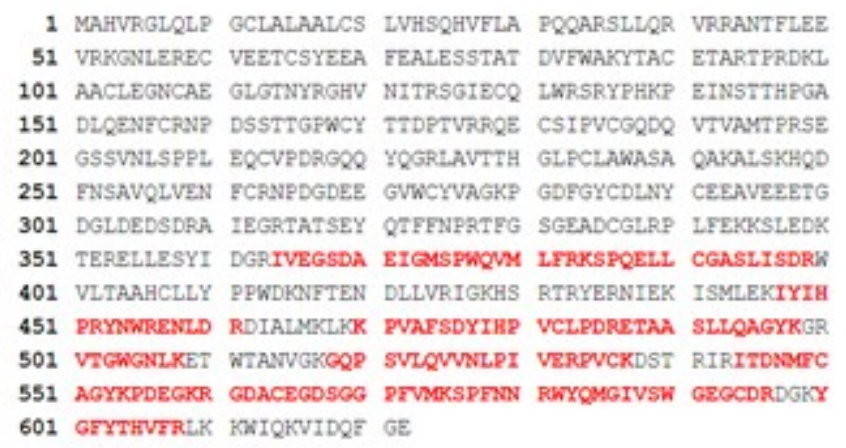

Spot 1Q, 2Q, 3Q. C-term fragment of THRB

\section{Figure 3}

Protein identification by Peptide Mass Fingerprint (PMF) of spots $1 \mathrm{Q}, 2 \mathrm{Q}$ and $3 \mathrm{Q}$ corresponding to Prothrombin. In particular, the identification highlights the C-fragment of thrombin (from aa 364 to 622 ) 
corresponding to thrombin heavy chain in UniProt database. (Figure magnification is part of the gel image in figure 2 "Reduced FT3").

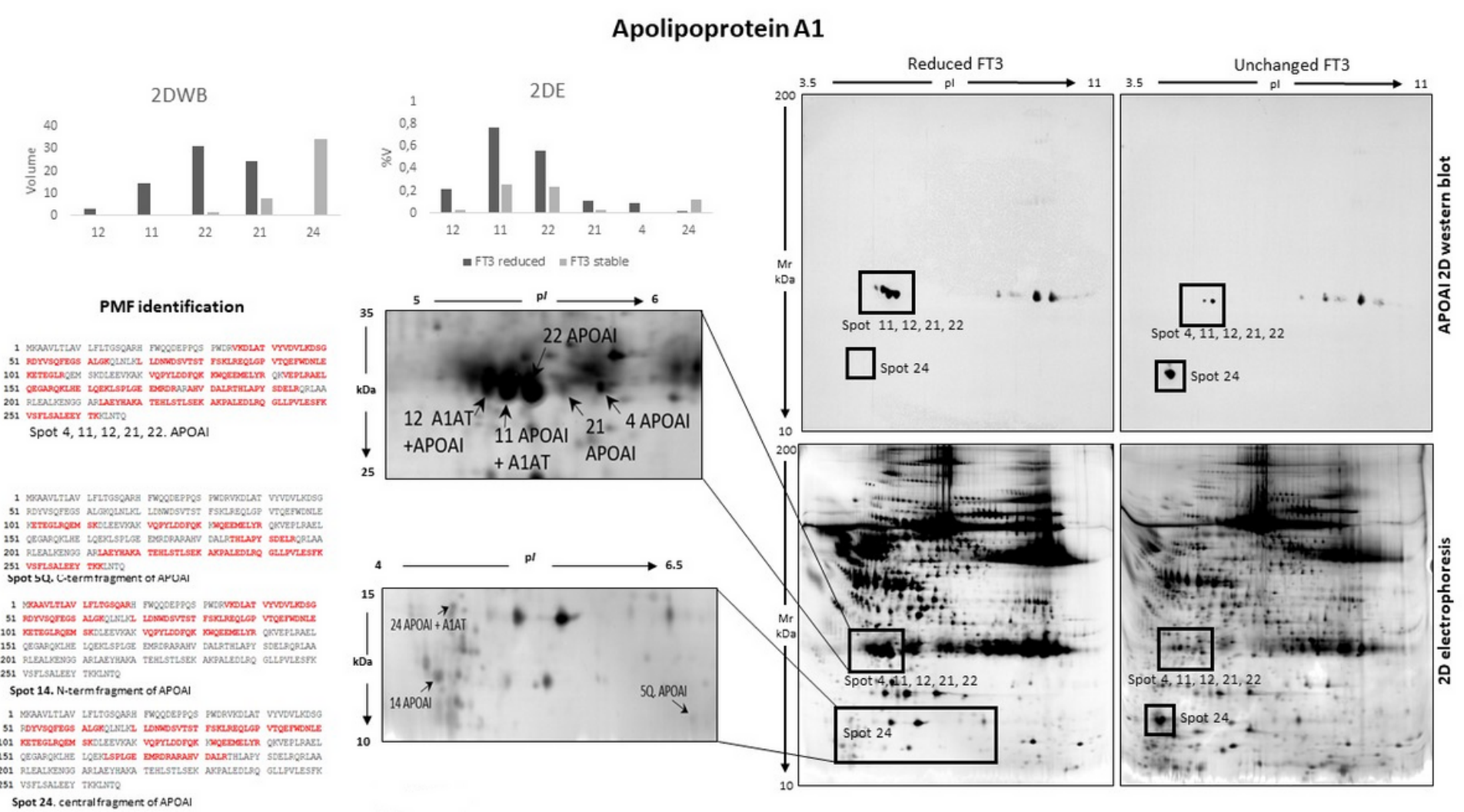

\section{Figure 4}

Protein identification by PMF of spots 4, 11, 12, 21, 22 as APOAl full-length and protein identification of spot $5 \mathrm{Q}$ as $\mathrm{C}$-terminal fragment, spots 24 as central fragment and 14 as $\mathrm{N}$-terminal fragment of APOAl. These spots are well visible on the 2D gel highlights. 2DWB with the antibody against-APOAI was also performed to visualize APOAl subproteome. 2DWB spot abundance expressed as volume, confirm the $2 \mathrm{DE}$ spot abundance expressed as percentage of relative volume $(\% \mathrm{~V})$. (2DWBs reported in figure are the entire WB membrane images). 

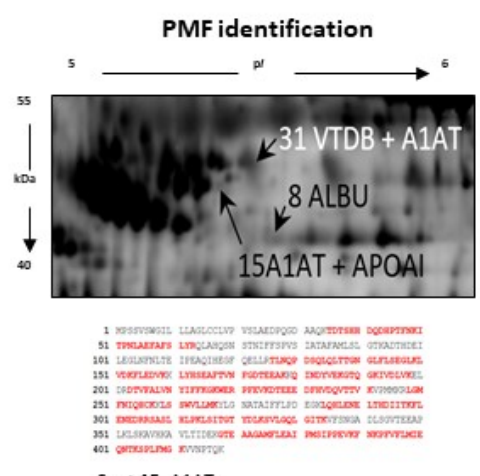

Spot 15. A1AT

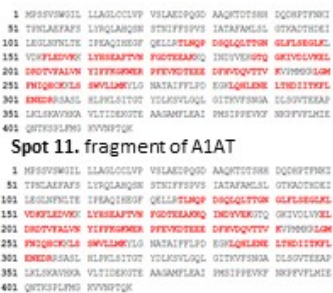

Spot 12 , fragment of A1AT

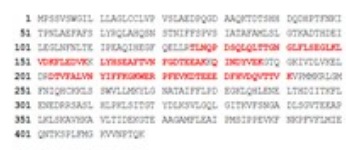

Spot 24. Smaller fragment of A1AT

\section{Alpha 1 antitrypsin}
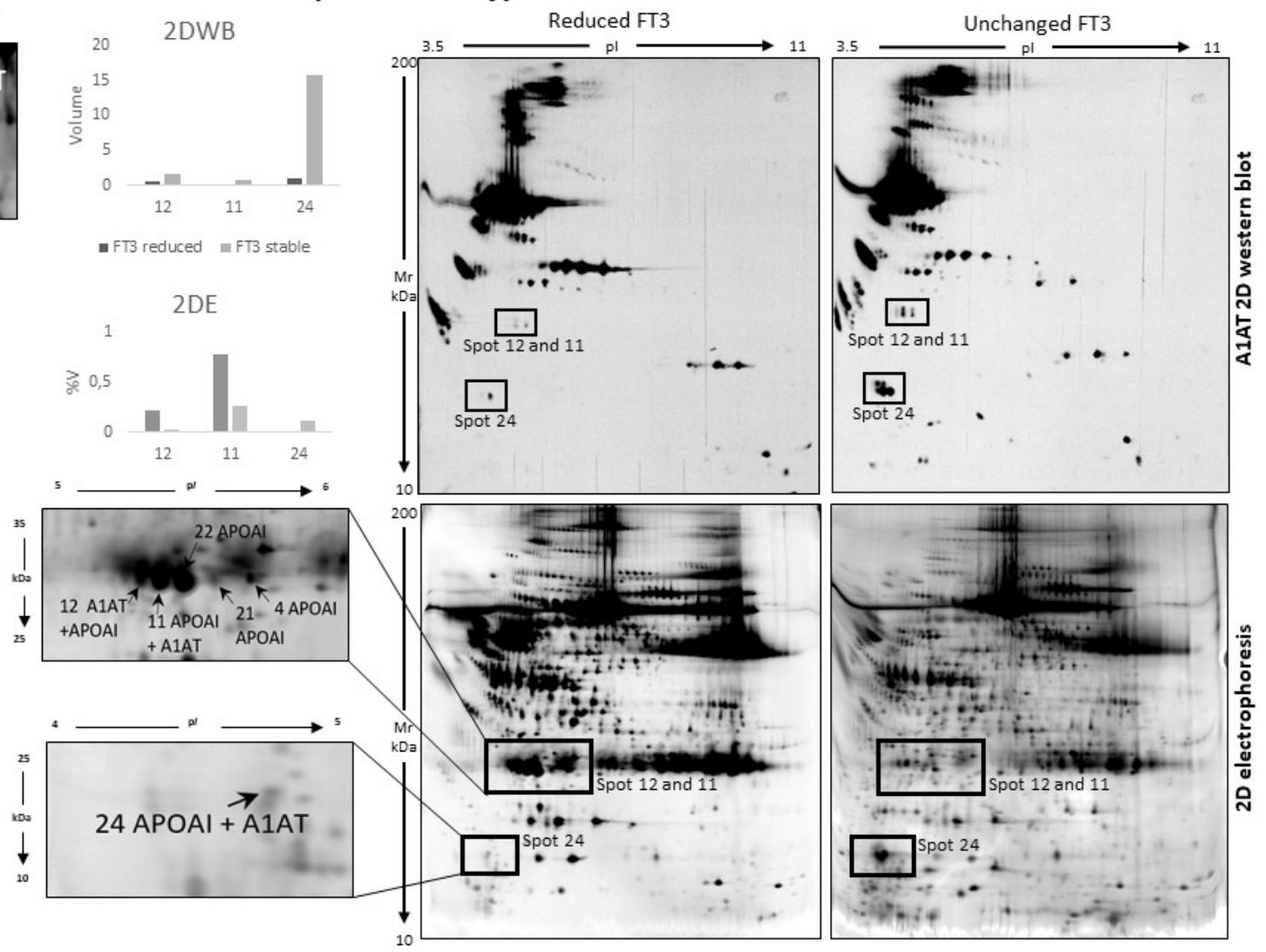

\section{Figure 5}

Protein identification by PMF of spot 15 corresponding to the A1AT full-length, while spots 12, 11, 24 identified central fragments of A1AT. These spots are well visible on the 2D gel highlights. 2DWB with the antibody against-A1AT was also performed to visualize A1AT subproteome. 2DWB abundance of spots 12 and 11 expressed as volume, shows an opposite behavior with respect to 2DE spot abundance expressed as percentage of relative volume (\%V). Instead, 2DWB abundance of spot 24 corresponds to 2DE abundance. (2DWBs reported in figure are the entire WB membrane images) 

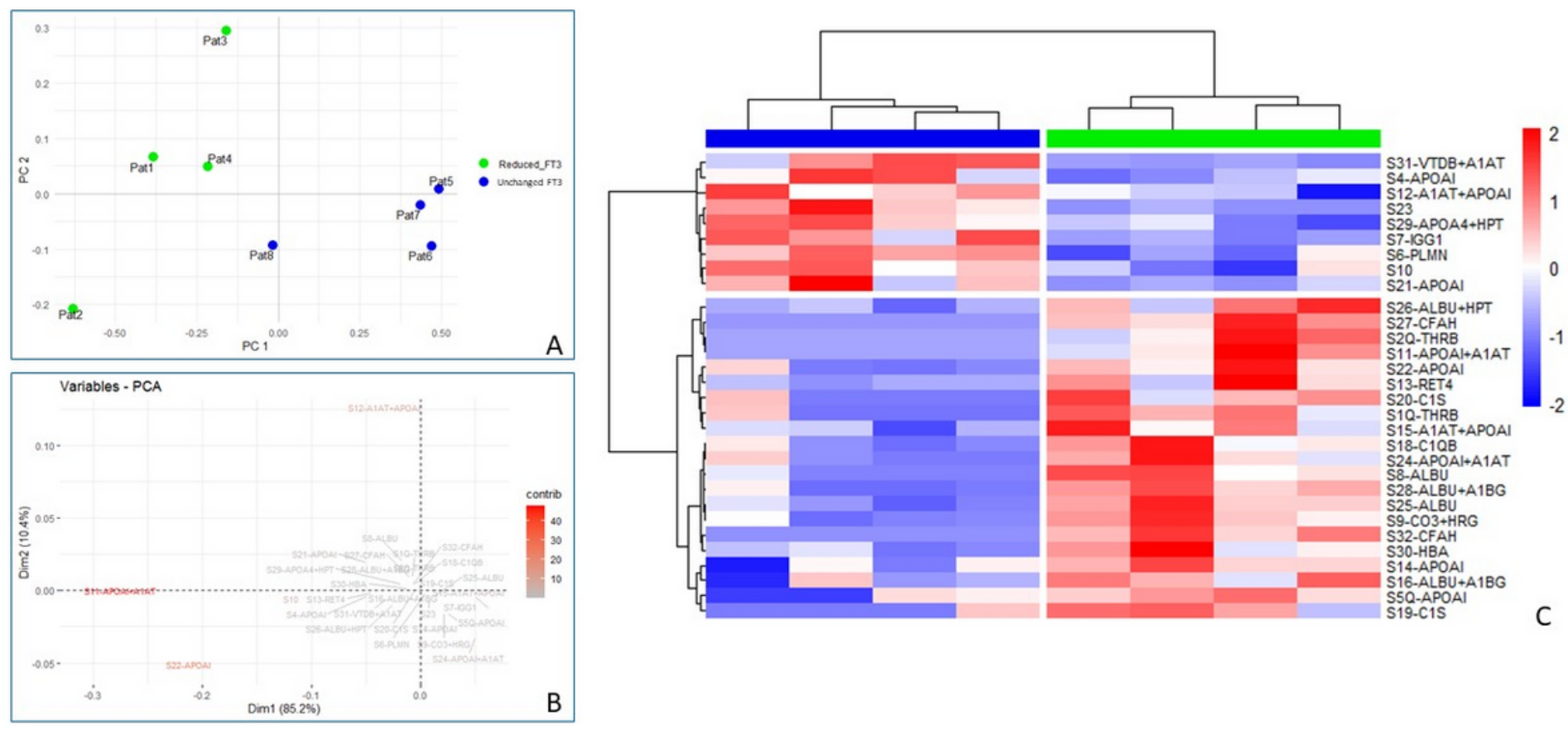

Figure 6

(A) PCA carried on the 31 DASs summarized the $95.6 \%$ (PC1:85.2\% and PC2:10.4\%) of the variance and the two groups clusterized alongside the first component. (B) PCA reporting the contributions of each significant variant in the first two PCs highlighting spot 11,12 and 22, identified as APOAI and fragments of A1AT, as the most significant. (C) Heatmap branching of the 31 DASs separates samples in two principal clusters corresponding to FT3 groups.

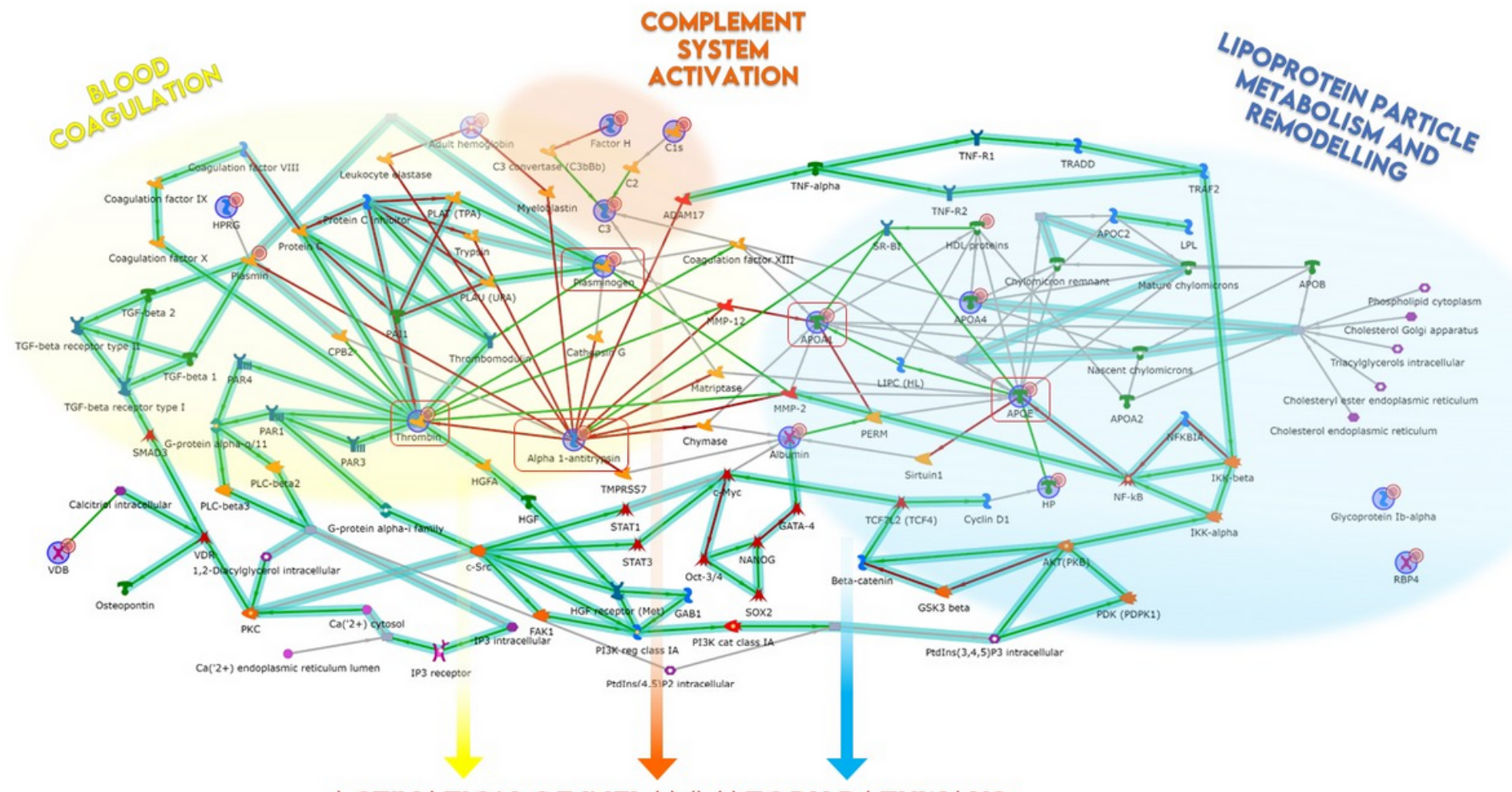

ACTIVATION OF INFLAMMATORY PATHWAYS 


\section{Figure 7}

Protein network built by MetaCore software uploading the 31 DASs. Thrombin, alpha1-antitrypsin, APOE, Plasminogen and APOA1 were central functional hubs, circled in red. Three principal molecular paths were circled by dashed lines respectively in yellow corresponding to blood coagulation, orange as complement system and blue as lipoprotein particle remodeling.

\section{Supplementary Files}

This is a list of supplementary files associated with this preprint. Click to download.

- Tables.docx

- SupplementaryFiles.docx 